\title{
RECUBRIMIENTOS DE VC Y NBC PRODUCIDOS POR DRT: TECNOLOGÍA ECONÓMICA, EFICIENTE Y AMBIENTALMENTE LIMPIA
}

\author{
VC AND NBC COATINGS PRODUCED BY TRD - AN ENVIRONMENTALLY CLEAN, INEXPENSIVE \\ AND EFFECTIVE TECHNOLOGY \\ Fabio Enrique Castillejo Nieto \\ Ing. Profesor Asistente de la Facultad de Ciencias de la Universidad Santo Tomas \\ Fabio_castillejo@usta.edu.co \\ Jhon Jairo Olaya Flórez \\ Ph.D. Profesor Asociado de la Facultad de Ingeniería de la Universidad Nacional de Colombia \\ jjolaya@unal.edu.co
}

Fecha de recepción: 25 de enero de 2012

Fecha de aprobación: 08 de mayo de 2012

\begin{abstract}
RESUMEN
En este artículo se presentan los fundamentos del proceso de deposición por difusión termoreactiva (DTR), una técnica que permite producir recubrimientos duros de forma económica sin ser invasiva o agresiva con el medio ambiente. Se propone una metodología experimental para desarrollar recubrimientos de carburos de vanadio (vc), y carburos de niobio (NDC), sobre aceros AIsı H13, utilizando en el proceso de fabricación DRT, bórax fundido, aluminio y ferroaleaciones a $1.020^{\circ} \mathrm{C}$ durante cuatro horas. Las muestras obtenidas fueron evaluadas utilizando difracción de rayos $X$ (DRX), para identificar las fases y microscopía electrónica de barrido (SEM), para medir el espesor y la homogeneidad de los recubrimientos producidos. La dureza fue obtenida usando microdureza knoop con una carga de $50 \mathrm{~g}$ y la resistencia a la corrosión fue determinada con técnicas electroquímicas, utilizando polarización potenciodímica. Los resultados confirmaron la presencia de recubrimientos de carburo de vanadio y carburo niobio con buena regularidad en el espesor, altas durezas y una mejora en la resistencia a la corrosión en comparación con el acero sin recubrimiento.
\end{abstract}

Palabras clave: NbC, termoreactiva, recubrimientos duros, vc.

\begin{abstract}
This paper offers basics of thermo-reactive deposition/diffusion process (TRD), a technique that may produce inexpensive and non-invasive or aggressive hard coatings to the environment. We
\end{abstract}


proposed an experimental methodology to develop coating of vanadium carbide (vc) and niobium carbide (NDC) on steel AISI H13. Coatings were deposited along with a mixed of aluminum, ferroalloys and borax at $1020^{\circ} \mathrm{C}$ for 4 hours. Samples were tested using X-ray diffraction (XRD) to identify the relevant phases and scanning electron microscopy (SEM) in order to measure thickness and homogeneity of coatings produced. Hardness status was obtained using Knoop microhardness with $50 \mathrm{~g}$ load, but corrosion resistance was measured by anodic polarization using dynamic power. Finally, results have verified the presence of niobium and vanadium carbide coating with good thickness homogeneity, higher hardness and corrosion resistance compared to uncoated steel.

Keywords: hard coating, NbC, thermo-reactive action, vc.

\section{INTRODUCCIÓN}

Si se utiliza baños de sales fundidas, aluminio y ferroaleaciones, es posible obtener recubrimientos que exhiban alta resistencia a la corrosión y al desgaste de una manera relativamente simple y a bajo costo [1]. Los recubrimientos se logran por inmersión de los materiales que van a ser tratados, en sales fundidas a elevadas temperaturas para alentar un proceso de difusión que al final, logra producir según la composición química de estos baños de sales, diferentes carburos, nitruros o boruros de metales. La difusión de elementos durante el proceso modifica la composición química de la superficie del material tratado y da lugar a la aparición de nuevas fases.

Ahora bien, las capas que se obtienen mediante el proceso DTR, tienen las mismas aplicaciones industriales que los recubrimientos de TiCN, CrN, y TiN que se obtienen con otras técnicas como la deposición física en fase vapor (PVD), o la deposición química en fase vapor (CVD) [2-4]. Sin embargo, dichas técnicas requieren condiciones de alto vacío, razón por la cual su implementación es mucho más costosa. La deposición por difusión termorreactiva es un proceso térmico utilizado para obtener recubrimientos de carburos de metales sobre aceros que contengan porcentajes de carbono mayores a $0.3 \%$ [1]. Estos recubrimientos presentan altas durezas, buena resistencia al desgaste, excelente adherencia al sustrato, buena resistencia a la corrosión y bajos coeficientes de fricción [3-4]. El proceso adopta la sencillez de los tratamientos termoquímicos tradicionales para conseguir capas de carburos de niobio, vanadio, titanio y cromo. Además, se puede encontrar recubrimientos homogéneos de alta dureza, con espesores superiores a 8 um, pero se puede encontrar recubrimientos con menos densidad que los fabricados con los procesos PVD y CVD.

Los recubrimientos obtenidos por DTR se producen en crisoles dentro de hornos de resistencia a altas temperaturas, no requieren algún tipo de atmosfera protectora y adicionalmente el proceso no emite ningún tipo de gases contaminantes [5,6]. El proceso de DTR se puede considerar como una combinación del tratamiento de borizado [7] y del tratamiento termoquímico de cementación. Cuando el sustrato se encuentra a altas temperaturas, el carbono disuelto en su 
matriz, se difunde hacia la superficie y queda preparado para combinarse químicamente con los elementos formadores de carburo presentes en el medio y se obtienen las capas superficiales de carburos. Esto sucede cuando las energías libres de formación del respectivo carburo son negativas, pero si esa energía es ligeramente negativa, la reacción llega rápido al equilibrio y por consiguiente, no se logra el recubrimiento deseado.

El ciclo típico para este tratamiento se muestra en la Figura 1. Antes de que las piezas sean procesadas, es importante hacer un precalentamiento con el fin de evitar distorsiones y reducir el tiempo del proceso. Se recomienda usar la temperatura de austenizacion del acero que se esté tratando, para poder realizar el proceso de temple en aire, sal o aceite inmediatamente después del tratamiento DTR y por último, un tratamiento térmico de revenido para ajustar la dureza del sustrato.

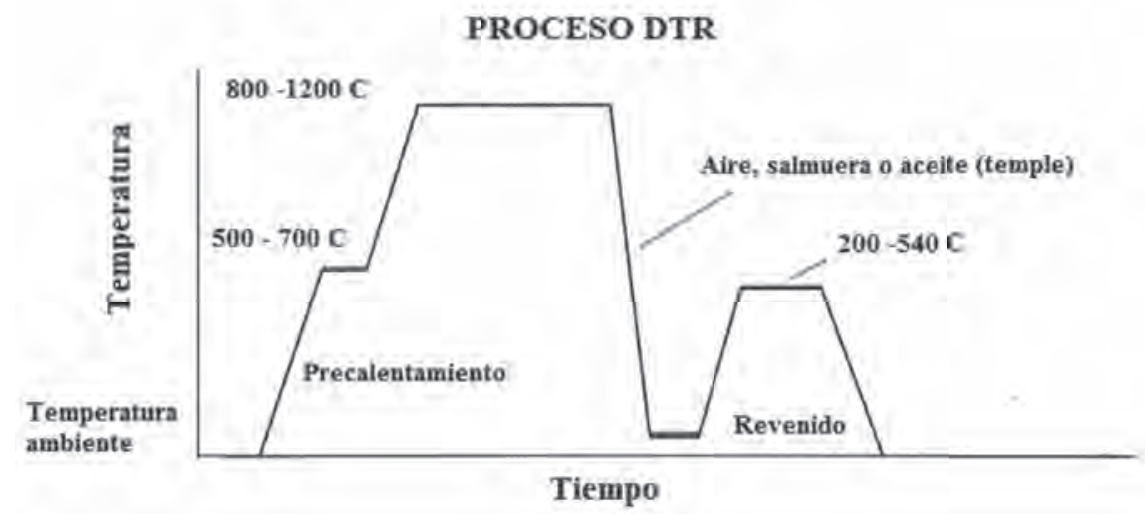

Figura 1. Ciclo tipico de tratamiento termico en el proceso DTR [1]

El proceso DTR se puede realizar en bórax fundido [8], en caja de polvo [9] o en lecho fluidizado [1], y mediante este proceso, se puede lograr recubrimientos de $\mathrm{VC}, \mathrm{NbC}, \mathrm{Cr}_{7} \mathrm{C}_{3}$ y $\mathrm{Cr}_{23} \mathrm{C}_{6}$ [1]. La capa se logra por la reacción química del carbono suministrado por el sustrato y los elementos formadores de carburos (EFC's), tales como el cromo, el vanadio, el niobio y el titanio [2]. En general, las capas de carburos se logran según los siguientes pasos:

- Se disuelve el elemento formador de carburo en el bórax que está contenido en la ferroaleación en polvo adicionada.

- El carbono libre en el acero (por lo general los investigadores trabajan con aceros de medio o alto carbono), se combina con el elemento formador de carburo para producir la capa superficial dura.

- La capa crece a medida que el carbono del substrato llega a la superficie para reaccionar con el elemento formador de carburo. 
- El espesor de la capa formada es independiente de la composición de la mezcla si los elementos formadores de carburo se añaden en suficiente cantidad, pero está relacionado con la temperatura del proceso, con el tiempo de inmersión, con la clase de material del substrato, el tipo de carburo y el tamaño del área que se va a recubrir.

Con el proceso DTR, se puede producir recubrimientos duros como el carburo de vanadio y el carburo de niobio que mejoran considerablemente las propiedades mecánicas de los aceros. Por ejemplo: Ios recubrimientos de VC aumentan notablemente la resistencia al desgaste de las piezas recubiertas en valores entre 9 y 30 veces mayor que las reportadas para aceros templados [10]. En esta investigación, se propuso una metodología experimental para el desarrollo de recubrimientos de carburos de vanadio (VC) y carburos de niobio (NDC), sobre aceros AISI H13, utilizando en el proceso de fabricación DRT, bórax fundido, aluminio y ferroaleaciones a $1020^{\circ} \mathrm{C}$ durante cuatro horas. Para ello, las muestras obtenidas fueron evaluadas, utilizando difracción de rayos X para identificar las fases, y microscopía electrónica de barrido para medir el espesor y la homogeneidad de los recubrimientos producidos. También se hizo una evaluación de la resistencia a la corrosión por medio de técnicas electroquímicas, utilizando polarización potenciodímica.

\section{PROCEDIMIENTO EXPERIMENTAL}

A continuación, se propone una metodología experimental para producir con éxito recubrimientos duros de VC y NDC. Estos recubrimientos fueron producidos sobre sustratos de acero para herramientas AISI H13. La preparación de las muestras consistió en un corte con geometrías de $15 \times 15$ × 4 mm y pulido hasta llegar a papel lija de 1.200, luego se realizó una limpieza ultrasónica en un baño de acetona. La composición química del acero se muestra en la Tabla 1.

Tabla 1. Composición química del acero AISI H13

\begin{tabular}{|c|c|c|c|c|c|}
\hline$\% \mathbf{C}$ & $\% \mathbf{C r}$ & $\% \mathbf{M n}$ & $\% \mathbf{S i}$ & $\% \mathbf{V}$ & $\% \mathrm{Fe}$ \\
\hline 0,38 & $4,9-5,3$ & $0,15-0,35$ & 1 & $0,6-0,9$ & Balance \\
\hline
\end{tabular}

Para producir los recubrimientos, se construyó un horno de resistencias que permite elevar la temperatura hasta $1.200^{\circ} \mathrm{C}$. El proceso de fabricación está basado en el calentamiento y fusión del bórax en un crisol de acero inoxidable, en donde se introducen los sustratos y se eleva la temperatura hasta $1020^{\circ} \mathrm{C}$. Finalmente se introduce aluminio y la ferroaleación de niobio o vanadio por un período de cuatro horas, para garantizar la formación del recubrimiento. En la Tabla 2, se presenta un resumen de las condiciones de depósito.

Se estudió la formación de fases en los recubrimientos, utilizando un difractómetro de rayos $X$ (XPert Panalytical), operando a 45 kV, 40 mA y con una radiación monocromatizada CuKa con 
una longitud de onda de 1,56 A. El tamaño de paso fue de $0,02^{\circ}$ con 40 s por paso. Se utilizó un microscopio electrónico de barrido ambiental marca Philips XL30 para caracterizar la sección transversal y la medición del espesor en diez puntos diferentes. Los valores de microdureza Knoop se obtuvieron, aplicando una carga de $50 \mathrm{~g}$ sobre el recubrimiento y tomando un promedio de 10 mediciones.

Tabla 2. Condiciones de los recubrimientos obtenidos

\begin{tabular}{|c|c|c|c|c|}
\hline RECUBRIMIENTO & COMPOSICIÓN QUÍMICA DEL BAÑO & ACERO & TEMPERATURA & TIEMPO \\
\hline VC & $16 \% \mathrm{Fe}-\mathrm{V}, 4,5 \% \mathrm{Al}$ y bórax & AISI H13 & $1020{ }^{\circ} \mathrm{C}$ & $4 \mathrm{~h}$ \\
\hline $\mathrm{NDC}$ & $16 \% \mathrm{Fe}-\mathrm{Nb}, 4,5 \% \mathrm{Al}$ y bórax & AISI H13 & $1020{ }^{\circ} \mathrm{C}$ & $4 \mathrm{~h}$ \\
\hline
\end{tabular}

Para evaluar la resistencia a la corrosión, se hicieron ensayos electroquímicos, utilizando las recomendaciones de la norma ASTM-G5, y se empleó un contraelectrodo de grafito de alta pureza y un electrodo de referencia de calomel saturado (SCE). El área de exposición de la muestra fue de $0.0706 \mathrm{~cm} 2$. Los experimentos se realizaron, variando el potencial entre $-0,3$ y $0.7 \mathrm{~V}$ con una velocidad de $30 \mathrm{mV} / \mathrm{min}$ después de 45 minutos de inmersión en un electrolito de $\mathrm{NaCl}$ al 3\% a temperatura ambiente. Se obtuvieron curvas de polarización potenciodinámica, usando un potencióstato-galvanostato marca GAMRY Instruments referente 600.

\section{RESULTADOS Y DISCUSIÓN}

Las Figuras 2 y 3 muestran los patrones de difracción de rayos x para los recubrimientos realizados. En el primer caso (Figura 2), se verificó la presencia de NbC en el recubrimiento según el patrón de referencia JCPDS 00-038-1364 con estructura cristalina cúbica. El patrón experimental tuvo una orientación mixta con picos de gran intensidad en las orientaciones (111), (200), (220) y (311), y de baja intensidad en las orientaciones (222), (400), (331), (420) y (422). En el segundo caso (Figura 3), se logró verificar la presencia de la fase VC en el recubrimiento de acuerdo con el patrón de referencia JCPDS 00-035-0786 con estructura cúbica. El patrón experimental mostró picos de alta intensidad en las orientaciones (222), (400), (440) y de baja intensidad en las orientaciones (1 10), (311), (330), (332), (642), (444), (550), (800), (662) y (840).

La producción de estos recubrimientos con una estructura cristalina, se puede atribuir al adecuado control de los parámetros termodinámicos que intervienen en el proceso de crecimiento. Por ejemplo: el caso específico de formación del VC se debe a la capacidad de un metal para reducir el bórax, lo cual se puede analizar mediante la reacción (1).

$$
\frac{2}{3} \mathrm{~B}_{2} \mathrm{O}_{3}+\mathrm{aM} \rightarrow \frac{4}{3} \mathrm{~B}+\mathrm{bM}_{\mathrm{x}} \mathrm{O}_{\mathrm{y}}
$$




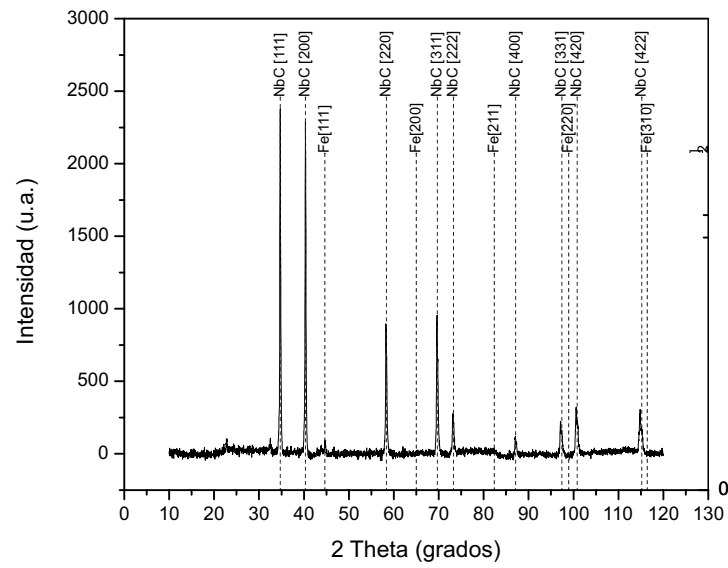

Figura 2. Patrón DRX para el recubrimiento de $\mathrm{NbC}$ sobre un acero AISI H13

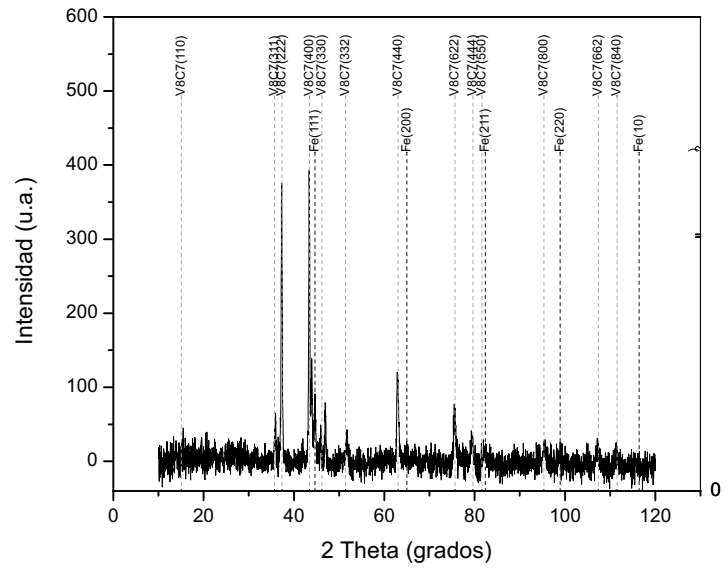

Figura 3. Patrón DRX para el recubrimiento VC Sobre un acero AISI H13

Donde $M$ representa el metal agregado al baño de bórax fundido. En los casos cuando esta reacción sea termodinámicamente posible a la temperatura de tratamiento, el bórax $\left(\mathrm{B}_{2} \mathrm{O}_{3}\right)$, se reduce y el metal agregado se oxida, por lo cual queda boro libre en la mezcla, y tiende a formarse sobre la superficie del material tratado, una capa de boruro de hierro (FeB, Fe $\mathrm{B}$ ). Cuando esta reacción no es termodinámicamente posible, el metal agregado queda libre de oxidación y se encuentra disponible para combinarse con el carbono del acero para formar el carburo.

En el proceso de formación del recubrimiento, la mezcla de bórax fundido y el polvo de aluminio se puede dar en un cambio de energía libre a $1.000^{\circ} \mathrm{C}(1.273 \mathrm{~K})$, mediante la reacción de reducción del $\mathrm{B}_{2} \mathrm{O}_{3}$, así:

$$
\begin{aligned}
& 2 / 3 \mathrm{~B}_{2} \mathrm{O}_{3}+4 / 3 \mathrm{Al} \longrightarrow 4 / 3 \mathrm{~B}+2 / 3 \mathrm{Al}_{2} \mathrm{O}_{3} \\
& \begin{array}{ccccc}
\Delta \mathrm{G}_{1273}(\mathrm{kcal}) & -154 & 0 & 0 & -203 \\
\Delta \mathrm{G}_{1273}=(0+(-203)) & -(-154+0)=-49 \mathrm{kcal}
\end{array}
\end{aligned}
$$

Por lo cual la reacción es termodinámicamente posible ( $\Delta \mathrm{G}<0)$, y se espera la reducción del $\mathrm{B}_{2} \mathrm{O}_{3}$ y la formación de capas de boruro. Ahora bien, para el caso específico, en la formación del carburo de vanadio, el polvo de ferrovanadio genera un cambio de energía libre a $1.000^{\circ} \mathrm{C}(1.273 \mathrm{~K})$ mediante la reacción de reducción del $\mathrm{B}_{2} \mathrm{O}_{3}$, así:

$$
\begin{array}{ccccc}
2 / 3 \mathrm{~B}_{2} \mathrm{O}_{3} & +4 / 3 & \mathrm{~V} & 4 / 3 \mathrm{~B} & 2 / 3 \mathrm{~V}_{2} \mathrm{O}_{3} \\
\Delta \mathrm{G}_{1273}(\mathrm{kcal}) & -154 & 0 & 0 & -146 \\
& \Delta \mathrm{G}_{1273}=(0+(-146)) & -(-154+0)=+8 \mathrm{kcal}
\end{array}
$$


Por lo cual la reacción no ocurrirá, y se espera que el vanadio libre en la mezcla, se combine con el carbono disuelto en la matriz del sustrato para formar carburo de vanadio $[11,12]$. Se puede observar en este caso, que el bórax $\left(\mathrm{B}_{2} \mathrm{O}_{3}\right)$, además de prestarse como medio donde ocurren las reacciones [13], está en capacidad de reducir el óxido del metal adicionado a la mezcla (reacción en sentido contrario). Esta característica colabora en la limpieza de las superficies por tratar y facilita la deposición de las capas de carburo.

Las Figuras 4a y 4b muestran las micrografías de la sección trasversal del acero AISI H13 tratado en los dos diferentes baños de sales, con ferroniobio y con ferrovanadio respectivamente. En ambos casos, se aprecia un recubrimiento homogéneo, compacto y adherido al sustrato. Para el caso del $\mathrm{NDC}$, presentó un espesor de 9,98 $\pm 0,26 \mu \mathrm{m}$ y el recubrimiento de VC, un espesor de 8,34 $021 \mu \mathrm{m}$. También se pudó notar que los recubrimientos presentan algunos poros.

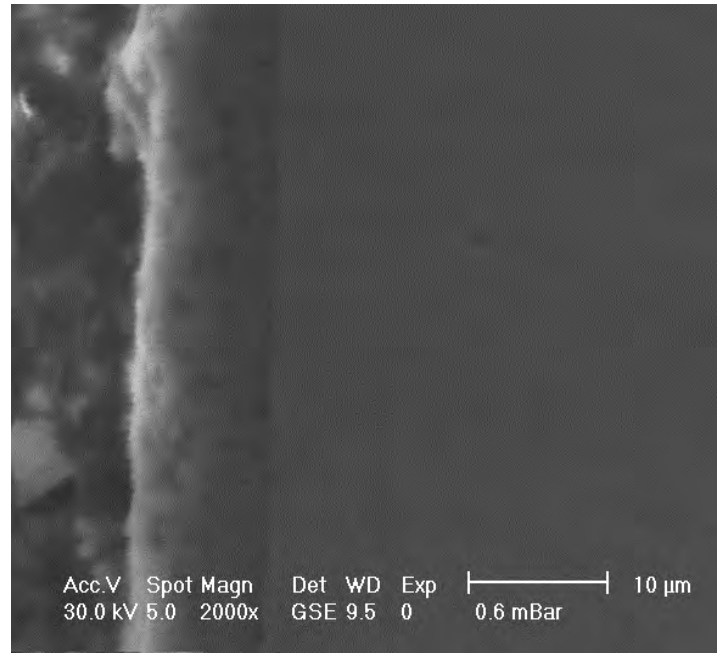

a) $\mathrm{NbC}$

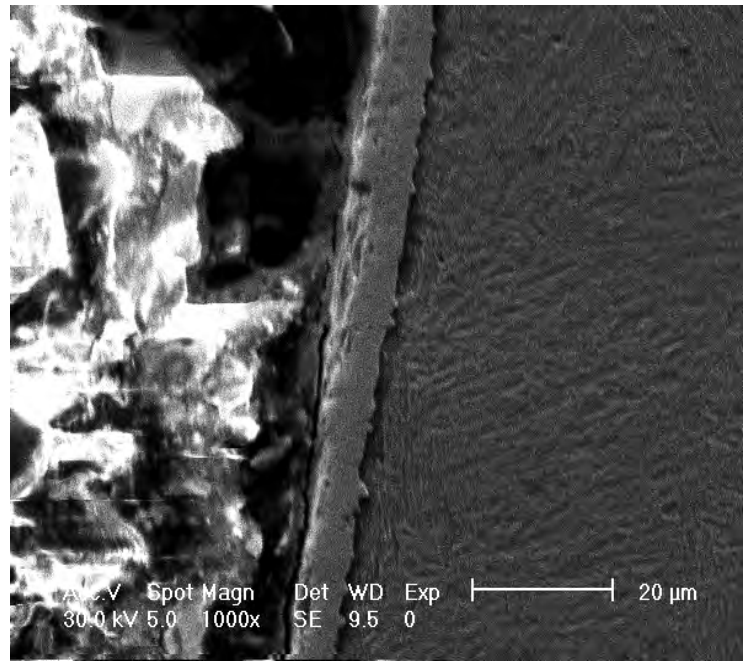

b) VC

Figura 4. Micrografías SEM del acero AISI H13 tratado en baño de sales con TRD.

a) carburo de niobio, b) carburo de vanadio

La microdureza de los recubrimientos evaluada en esta sección transversal, fue de $2197.9 \pm 46.88$ HK para el VC y de $2180.5 \pm 25.5$ HK para el NbC. Estos valores de microdureza se encuentran dentro del rango esperado para este tipo de recubrimientos $[14,15]$.

La microestructura de los recubrimientos producidos con el sistema DTR, depende de la movilidad superficial de los átomos de carbono en la superficie del sustrato, la cual se controla con los parámetros de depósito [16] y las propiedades químicas del sustrato [17]. Dependiendo del valor de la actividad del carbono en la superficie, se puede encontrar diferentes morfologías que seguramente modificarán el desempeño mecánico y la resistencia a la corrosión del recubrimiento. 
La microestructura final del recubrimiento se determinó en función de la tasa de crecimiento perpendicular $V_{1}$ y horizontal $V_{h^{\prime}}$ al sustrato y las actividades del carbono [17].

Se puede lograr una microestructura de granos alargados cuando el recubrimiento crece sobre un sustrato con bajos valores en su actividad de carbono que favorece la disminución de la difusión de los átomos de carbono en la superficie de crecimiento. En estas condiciones físicas, se produce una baja tasa de nucleación que dificulta el crecimiento continuo del recubrimiento. Este modelo se ajusta bien cuando la velocidad de crecimiento horizontal del recubrimiento es alta y la velocidad de crecimiento vertical es inhibida debido a la baja movilidad o difusión de los átomos de carbono. También se puede lograr una microestructura de granos equiaxiales que se desarrolla en sustratos con valores de actividades de carbono suficiente para que la velocidad de crecimiento perpendicular sea aproximadamente igual a la velocidad de crecimiento horizontal. De esta forma, se favorece un aumento en la densidad de nucleación que permite disminuir el tamaño de grano y la formación de una microestructura compacta.

Los recubrimientos producidos en esta investigación, se ajustan bien a este modelo porque el acero AISI H13 tiene altos valores de actividad de carbono [17]. Por último, una tercera microestructura puede ser columnar, se desarrolla en sustratos con altos valores de actividad de carbono. En este caso, la tasa de crecimiento perpendicular es mayor que la tasa de crecimiento horizontal.

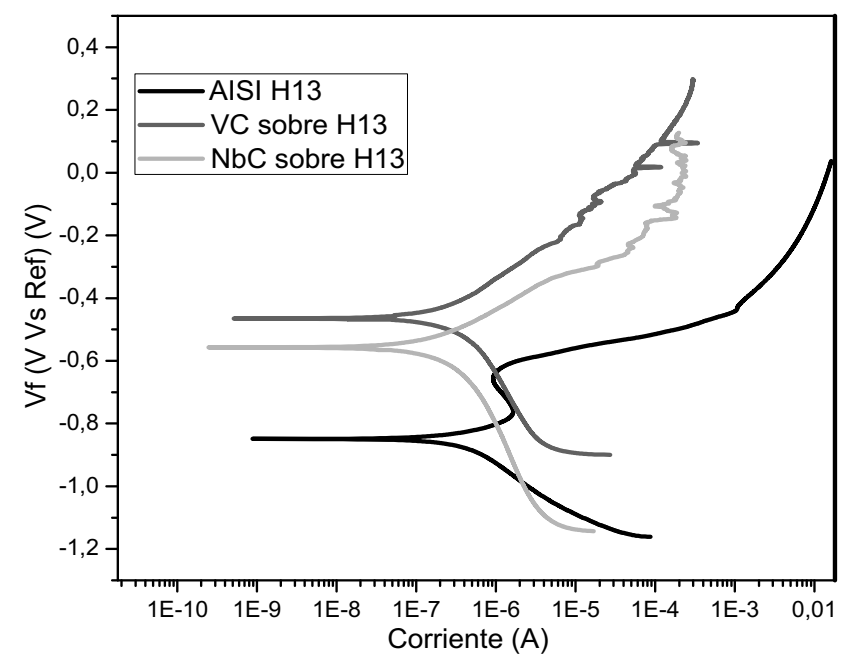

Figura 5. Curvas Tafel para el acero AISI H13 sin recubrimiento y con recubrimientos de $\mathrm{VC}$ y $\mathrm{NbC}$

La Figura 5 muestra los resultados de la polarización potenciodinámica (Tafel), para el acero sin algún recubrimiento y los recubrimientos de carburo de niobio y carburo de vanadio. En general, se logró verificar una mejor resistencia a la corrosión en los recubrimientos, considerando que se logró un aumento positivo del potencial de corrosión y la corriente de corrosión disminuyó aproximadamente un orden de magnitud. 
Es importante mencionar que los recubrimientos cerámicos pueden lograr potencias con valores positivos y menores densidades de densidad de corriente de corrosión. Sin embargo, se debe tener en cuenta la porosidad inherente y los limites de grano que se generan durante el crecimiento del recubrimiento. Estos defectos generan un par galvánico que se favorece como consecuencia de la diferencia del valor de Ecorr entre el recubrimiento y el acero. A través de dichos defectos, la solución corrosiva se difunde hacia el sustrato para que se corroa la interfase recubrimiento sustrato y la pérdida de adherencia. Se debe considerar que gran número de iones de Cl presentes en la sección transversal del recubrimiento, desde la superficie del recubrimiento hasta la interfase, promueven la reacción del sustrato con la solución de $\mathrm{NaCl}$ al 3\%. De esta forma, se va a generar una reacción catódica que ocurre en la superficie del recubrimiento, y una reacción anódica en la superficie del sustrato, descritas por las siguientes reacciones:

$$
\begin{array}{ll}
\mathrm{M}+\mathrm{ne} \rightarrow \mathrm{M}^{\mathrm{n+}} & \text { (Anódica) } \\
\mathrm{O}_{2}+2 \mathrm{H}_{2}+4 \mathrm{e} \rightarrow 4 \mathrm{OH}^{-} & \text {(Catódica) }
\end{array}
$$

A su vez, el proceso de corrosión se complementa con los iones producidos por la reacción anódica que se mueven a través de los poros del recubrimiento y se combinan con los productos $\mathrm{OH}$ - producidos en la reacción catódica, tal como lo describe la siguiente reacción:

$$
\mathrm{M}^{\mathrm{n}+}+\mathrm{nOH}^{-} \rightarrow \mathrm{M}(\mathrm{OH})_{\mathrm{n}}
$$

Sin embargo, sobre la superficie del sustrato puede ocurrir una reacción con los iones positivos que están presentes en la zona anódica, y con los iones de Cl- para mantener la neutralidad eléctrica. Por lo tanto, grandes cantidades de MCln se forman en la interfase, siguiendo la siguiente reacción:

$$
\mathrm{MCl}_{n}^{\prime}+\mathrm{nH}_{2} \mathrm{O} \rightarrow \mathrm{M}(\mathrm{OH})_{\mathrm{n}}+\mathrm{nHCl}
$$

Tanto los iones de $\mathrm{H}+$ como de $\mathrm{Cl}$ - que se difunden hasta el sustrato a través de los poros o las fronteras de los granos, pueden acelerar el proceso de corrosión en el sustrato, mientras que los productos de corrosión se van acumulando cada vez más, en los defectos del recubrimiento. Es así, como se aumentan las fuerzas de tensión hasta producir una delaminación del recubrimiento, y producen un proceso de corrosión preferencial del sustrato bajo el recubrimiento.

\section{CONCLUSIONES}

Por medio de la técnica de deposición por difusión termorreactiva, se logró depositar con éxito recubrimientos duros de NDC y VC sobre aceros AISI H13, con una buena homogeneidad y durezas superiores a $2.000 \mathrm{HK}$. 
El mecanismo de corrosión para los recubrimientos producidos seguramente ocurre a través de los poros y defectos del material, permitiendo que el electrolito penetre hasta llegar al sustrato; de esta forma, se genera corrosión localizada o reducción de la adherencia del recubrimiento.

El proceso DTR proporciona una nueva alternativa para producir carburos de metales de transición de forma económica, lo cual podría proporciona una posibilidad de desarrollo en Colombia, con la incorporación de esta tecnología en las empresas de tratamientos térmicos o superficiales.

\section{AGRADECIMIENTOS}

Los autores agradecen al doctor Rogelio Ospina y al laboratorio de física del plasma de la Universidad Nacional de Colombia sede Manizales, por la toma de las imágenes SEM. Además, a COLCIENCIAS por el apoyo económico mediante el contrato RC 338-2011

\section{REFERENCIAS BIBLIOGRÁFICAS}

[1] Aray T., and Harper S., (1991). ASM Handbook, ASM International, materials Park, OH, USA. Vol. 4, 448 p.

[2] Arai T., (1979). Carbide Coating Process by Use of Molten Borax Bath in Japan. In: J. Heat Treat. Vol. 1, pp. 15-22.

[3] Arai et al., (1988). Vanadium Carbonitride Coating by Immersing into Low Temperature Salt Bath, in Heat Treatment and Surface Engineering. George Krauss, Ed., ASM International. pp. 49-53.

[4] Shiming Hao et al., (1989). Acta Metall. Sin. Vol. 25, pp. B151- B155.

[5] Arai et al., (1987). Diffusion Carbide Coatings Formed in Molten Borax Systems. In: J. Mater. Eng, Vol. 9, pp. 183-189.

[6] Arai T., (1992). Tool materials and surface treat- ments. In: J. Mater. Proc. Technol. Vol. 35, pp. 515- 528.

[7] Campos I., Torres R., Ramírez G., Ganem R., and Martínez J., (2006). Growth kinetics of iron boride layers: Dimensional analysis. In: Applied Surface Science. Vol. 252, pp. 8662-8667.

[8] Oliveira et al., (2006). Evaluation of hard coatings obtained on AISI D2 steel by thermoreactive deposition treatment. Vol. 201, pp. 1880 -1885. 
[9] Sen U., (2004). Kinetics of niobium carbide coating produced on AISI 1040 steel by thermoreactive deposition technique. In: Materials Chemistry and Physics. Vol. 86, pp. 189-194.

[10] Wang G.B., (1997). Wear mechanisms in vanadium carbide coated steels, Wear. Vol. 212, pp. 25-32.

[1 1] Arai Komatsu T., and Mizutani K., (1975). Journal of the Japan Institute of Metals. Vol. 39, pp. 247-255.

[12] Mizutani K., (1977). Journal of the Japan Institute of Metals. Vol. 41, pp. 68-72.

[13] Mizutani K., (1977). Journal of the Japan Institute of Metals. Vol. 28 (2), pp. 35-39.

[14] Oliveira C.K.N., and Casteletti L.C , (2005). Surf. Eng. Vol. 21, 320 p.

[15] Subramanian C., Strafford K.N., Wilks T.P., and Ward L.P., (1999). On the desing of coating systems: Metallurgical and others considerations. In: J. Mater. Process. Technol. Vol. 56, pp. 385-397.

[16] Arai T., and Moriyama S., (1995). Growth behavior of chromium carbide and niobium carbide Layers on steel substrate, obtained by salt bath immersion coating process, Thin Solid Films. Vol. 259, pp. 174-180.

[17] Fana X.S., Yanga Y.G., Xiaa Z.X., Zhanga C., and Cheb H.Q., (2010). The microstructure evolution of VC coatings on AISI H13 and 9Cr18 steel by thermo-reactive deposition process. In: Journal of Alloys and Compounds. Vol. 505, pp. L15-L18. 
